\title{
Tunable IR/THz source for pump probe experiments at the European XFEL
}

E.A. Schneidmiller and M.V. Yurkov (DESY, Hamburg)

M. Krasilnikov and F. Stephan (DESY Zeuthen)

M. Izquierdo (European XFEL GmbH, Hamburg)

M. Gensch (HZDR, Rossendorf) 


\section{European XFEL}
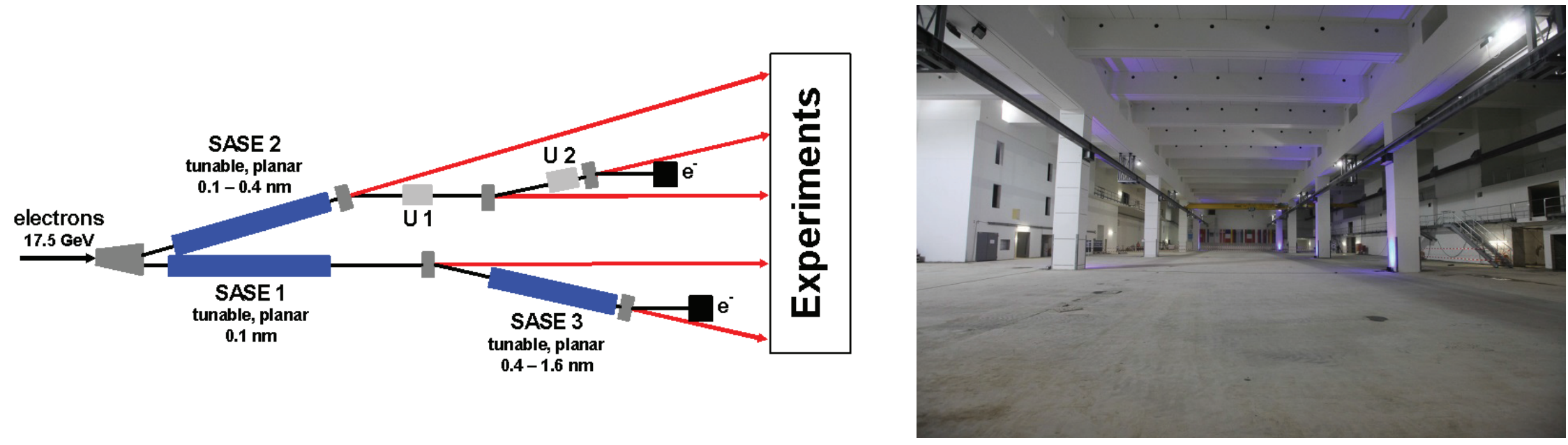

\begin{tabular}{lll}
\hline Location & Full length & Undulator \\
& & \\
\hline XS1-XS3 & $620 \mathrm{~m}$ & SASE1 \\
XS3-XHDU1 & $301 \mathrm{~m}$ & SASE3 \\
\hline & & \\
XS1-XS2 & $550 \mathrm{~m}$ & SASE2 \\
XS2-XS4 & $190 \mathrm{~m}$ & Spont. U1 \\
XS4-XHDU2 & $250 \mathrm{~m}$ & Spont. U2 \\
\hline
\end{tabular}

Baseline option:

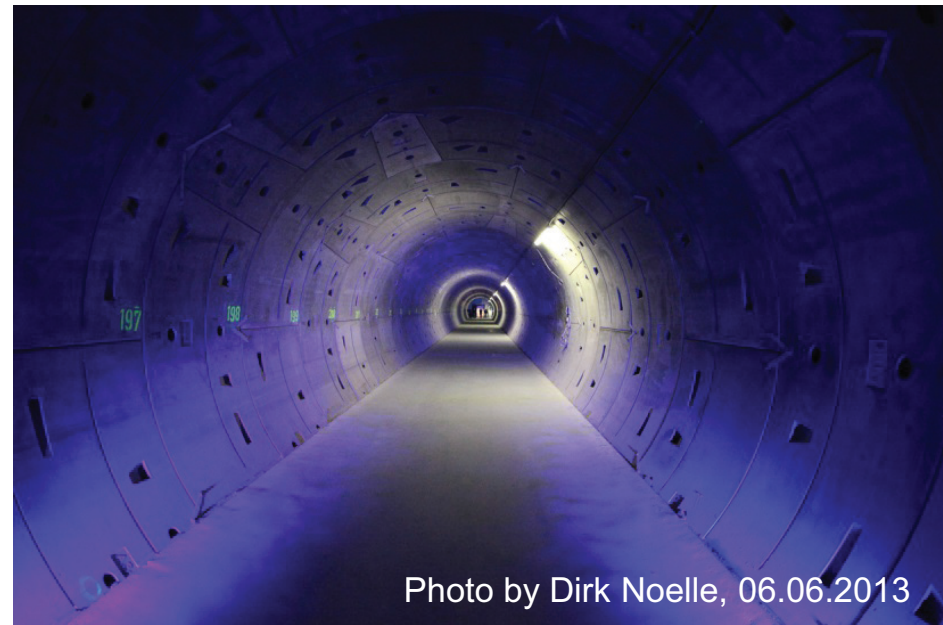

-3 FEL beamlines (SASE1/2/3) cover $0.05 \mathrm{~nm}-5 \mathrm{~nm}$ wavelength range

-Burst mode of operation: $10 \times 0.6$ ms pulse trains per second, 2700 pulses per train

-Laser lab is foreseen for pump-probe experiments (FEL+Laser) 
In this talk we briefly discuss problems of potential extensions of the European XFEL for pump-probe experiments with longer wavelengths: IR $\rightarrow \mathrm{FIR} \rightarrow \mathrm{THz}$ :

- Generation of IR/THz radiation with electron beams.

- IR/THz radiation source in the tunnel

- IR/THz radiation source driven by separate linac

Nothing is decided at this stage, since the subject of the presentation is beyond the baseline option.

Materials and opinions presented here reflect personal view of the authors. 
Scientific case is described in XFEL TDR. An activity on revision / extension of the scientific case using pump-probe techniques is in the progress:

-the study of protein dynamical transitions and tertiary native proteins with structural motions -the characterization of ions and molecules where solvation process plays a relevant role in the modification of their structure and properties.

-Condensed matter physics: the study of non-linear effects aiming to the control the state of material which could lead to new applications.

-Phase change of materials.

-Highly correlated materials (magnetoresistance, ferroelectrocity, superconductivity, insulator-tometal transitions, etc). 


\section{Pump-probe experiments at x-ray FEL user facility}

General requirements to the pump source:

-Time structure of IR/THz source must follow time structure of operation of x-ray pulses.

-IR/THz source should have wide tunability range .

-IR/THz source should provide wide possibilities for generation of different temporal and spectral patterns, polarization. For instance, some applications require strong single-cycle pulses. There is rather big amount of applications requesting narrow band radiation. Thus, different radiators are required.

-Many applications require strong peak power (field strength) or high pulse energy.

-Time jitter of pump and probe pulses should be small enough for resolving time-dependent phenomena.

Specific for the European XFEL:

-The European XFEL operates in the burst mode: $10 \times 0.6$ ms pulse trains per second, 2700 pulses per train (4.5 $\mathrm{MHz}$ rep. rate).

-Current wavelength range of interest spans from 6 microns ( $50 \mathrm{THz}, 0.206 \mathrm{eV}$ ) to 1000 microns (0.3 THz, $1.2 \mathrm{meV})$.

- Recalculation of user's requirements in terms of pulse energy spans a lot: microjoules $\rightarrow$ hundreds of microjoules $\rightarrow$ millijoules) .

-Time jitter: there are two types of experiments: (i) field driven dynamics where temporal resolution should be a few fs; (ii) "intensity" driven dynamics where temporal resolution is given by the longest pulse (e.g. if THZ pulse is 3 ps than timing only need to be 3 ps). 
- Here we discuss only accelerator based radiation sources. Attractive features are: clean production of the radiation in vacuum, tunability of radiation, potential to provide high power (high field), polarization control.

- Radiation occurs due to rearrangement of the field of electrons which happens when electrons are accelerated, pass boundary of media, pass trough an aperture:

- Radiation in bend magnet.

- Undulator radiation.

- Transition radiation (i.e., crossing metallic foil).

- Diffraction radiation (i.e. passing an aperture).

- Edge radiation

- Coherent enhancement of the radiation intensity occurs for short or tailored electron bunches.

Radiation power, averaged over an ensemble of $N$ electrons:

$$
\langle P(\omega)\rangle=p(\omega)\left[N+N(N-1)|\bar{F}(\omega)|^{2}\right],
$$

Radiation power of single electron is $p(\omega)$, and bunch form factor is: $F(\omega)=\frac{1}{N} \Sigma e^{i \omega t_{k}}$

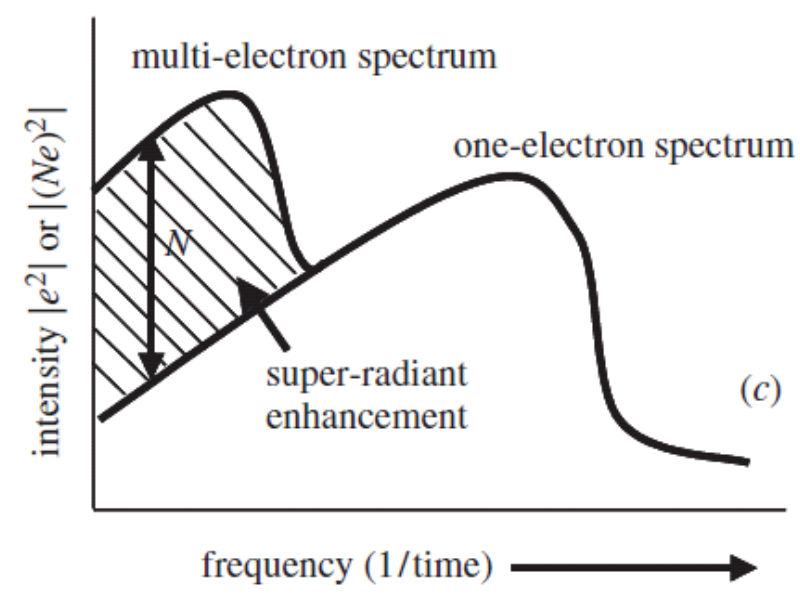




\section{Coherent radiation of electron beams}

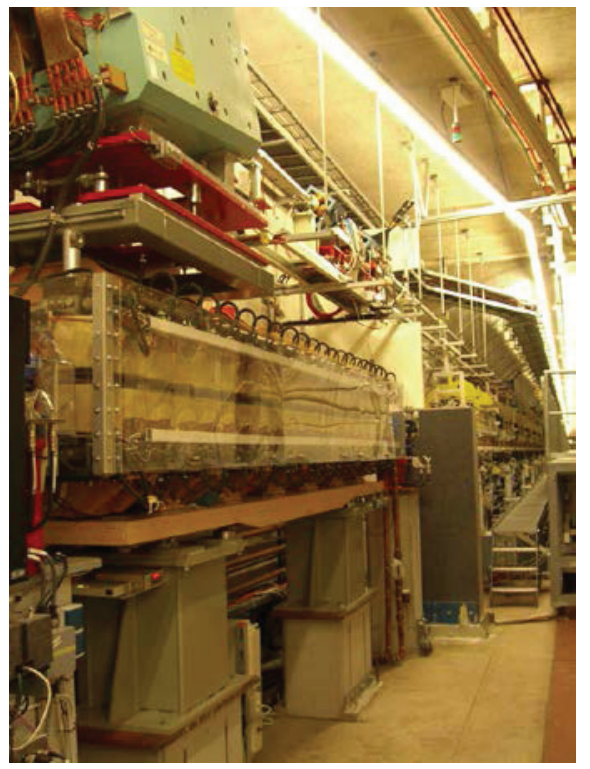

FIR coherent source at FLASH (DESY/HHU/JINR): THz pulse energies are in the range of $100 \mathrm{uJ}$

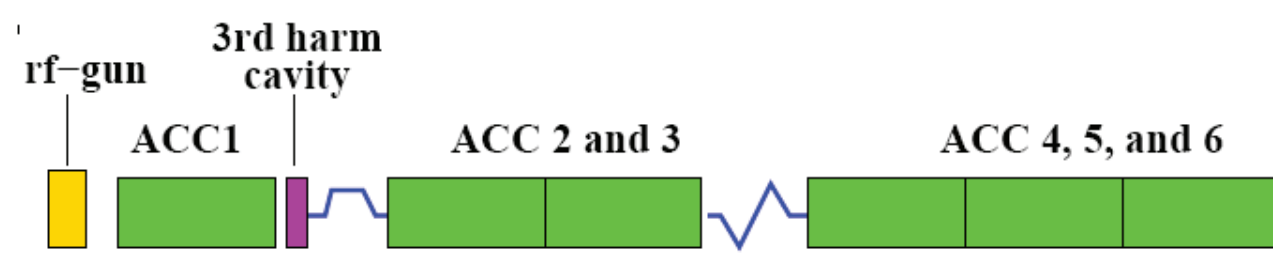

BC1
$\mathrm{BC} 2$

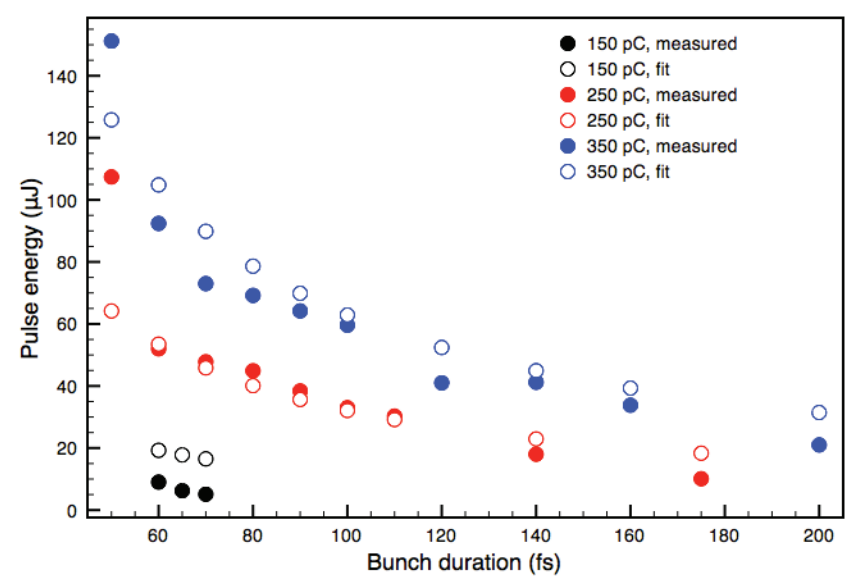

SLAC: Highly compressed, relativistic electron bunches are fired through a thin Be foil to generate intense, broadband, high-field $\mathrm{THz}$ transients. Pulse energies greater than $100 \mu \mathrm{J}$ and field strengths greater than $10 \mathrm{MV} / \mathrm{cm}$ are measured.

D. Daranciang et al., OSA/ CLEO 2011

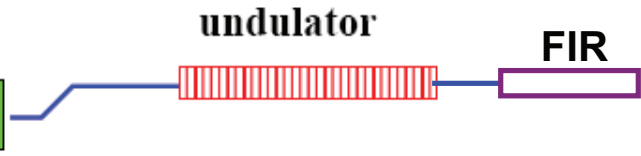

DL

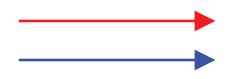

To pump-probe experiment 


\section{Pump-probe experiments at $\mathrm{x}$-ray FEL user facility}

- Generation of IR/THz pulse by the same electron beam after exit of the main undulator. Natural synchronization at high (fs) level can be achieved. Electron beam power of the European XFEL is in sub-MW level of average power. For this (ultimate) mode of operation only radiators which are not intercepting electron beam can be installed in the tunnel.

- Generation of bend radiation, or edge radiation is performed in a natural way in the electron beamline. Screens producing CTR/CDR can be used at a reduced electron beam current. These techniques provides single cycled pulses.

- Generation of relatively narrow band IR/THz radiation can be performed in the undulator as it is realized presently at FLASH. High electron beam energy requires to increase substantionally period and field. Recently we discussed this option with our colleagues from Budker Institute (Novosibirsk). It is technically feasible to install superconducting wiggler in the tunnel which will cover significant fraction of $\mathrm{THz}$ band at $17.5 \mathrm{GeV}$ nominal energy of electron beam.

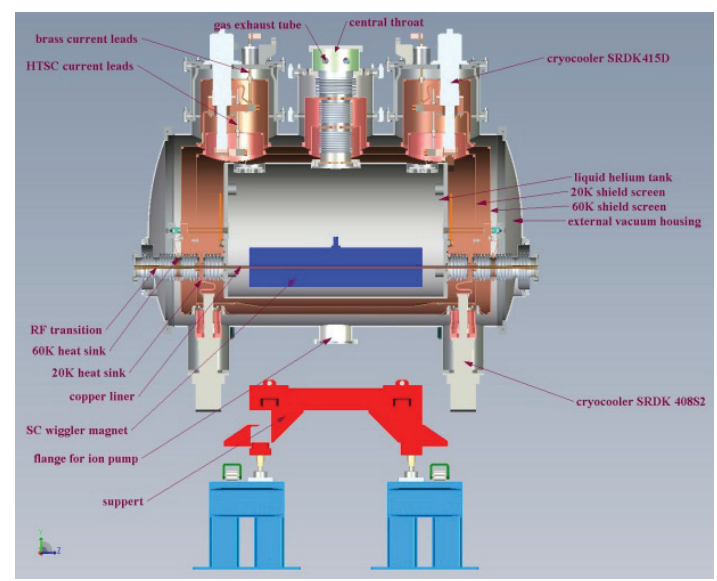

8T SC wiggler

(courtesy to N. Mezentsev and K Zolotorev, BINP)

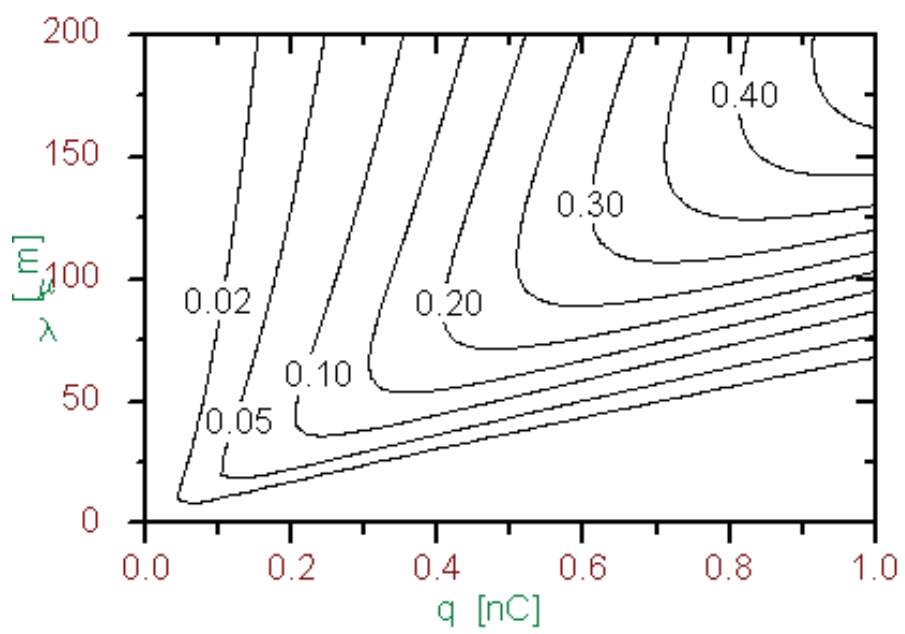

Pulse energy [mJ] versus wavelength and bunch charge 


\section{Pump-probe experiments at $\mathrm{x}$-ray FEL user facility}

HеLмноLтz

"2 in 1" approach solves of the time jitter problem, but creates a set of different problems:

-Transport of radiation at long distances ( a few hundreds meters): losses in the beamline, limited transmission band. An attempt to reduce diffraction losses at longer $(\mathrm{THz})$ wavelength will result in non-practical transverse dimensions of mirror-based optical beamline. Technical solution based on diaphragm focusing line has also limited frequency band.

-Technical difficulties to manipulate with high energy (8.5 GeV to $17.5 \mathrm{GeV})$ electron beams to produce different features of the radiation: specific temporal and spectral profiles, wavelength, polarization, peak and average power.

-Experience at FLASH shows that it is rather tricky task to provide good lasing and strong $\mathrm{THz}$ radiation from the same bunch. Two-bunch schemes were discussed where the first bunch generates IR/THz radiation, but does not lase. Background from the first bunch passing the undulator is the subject of concern. One of the solutions under discussion at LCLS is directing of the first bunch via by-pass - significant technical complication.

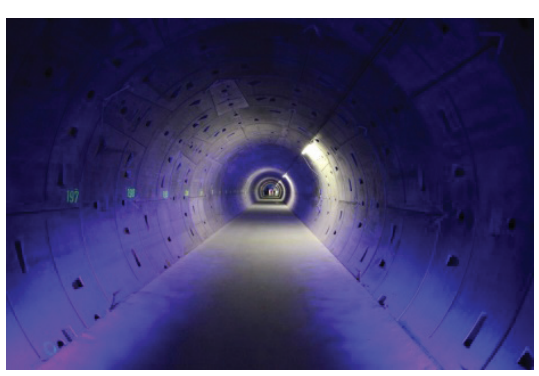

\begin{tabular}{lll}
\hline Location & Full length & Undulator \\
\hline XS1-XS3 & $620 \mathrm{~m}$ & SASE1 \\
XS3-XHDU1 & $301 \mathrm{~m}$ & SASE3 \\
\hline & & \\
XS1-XS2 & $550 \mathrm{~m}$ & SASE2 \\
XS2-XS4 & $190 \mathrm{~m}$ & Spont. U1 \\
XS4-XHDU2 & $250 \mathrm{~m}$ & Spont. U2 \\
\hline
\end{tabular}

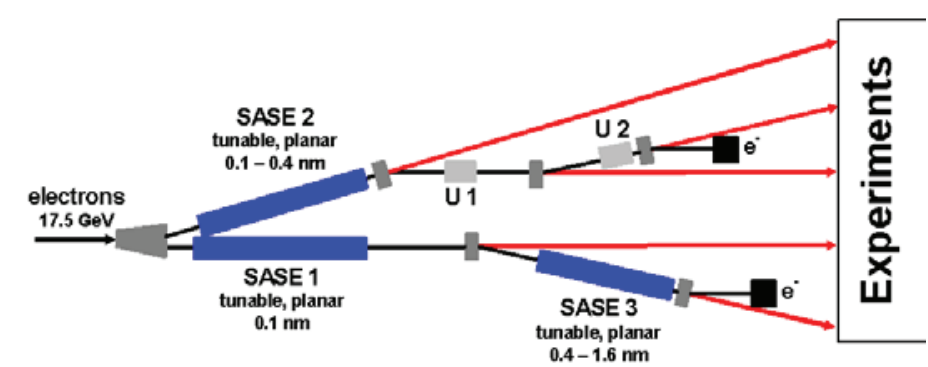




\section{Pump-probe experiments at $\mathrm{x}$-ray FEL user facility}

- For low repetition rate one can use coherent transition radiators. This technical solution is under realization at LCLS. Sample will be placed in the tunnel due to difficulties with $\mathrm{THz}$ beam transport to experimental area.

THz/X-ray Pump Probe (THXXP) ${ }_{\text {A. Fisher }}$

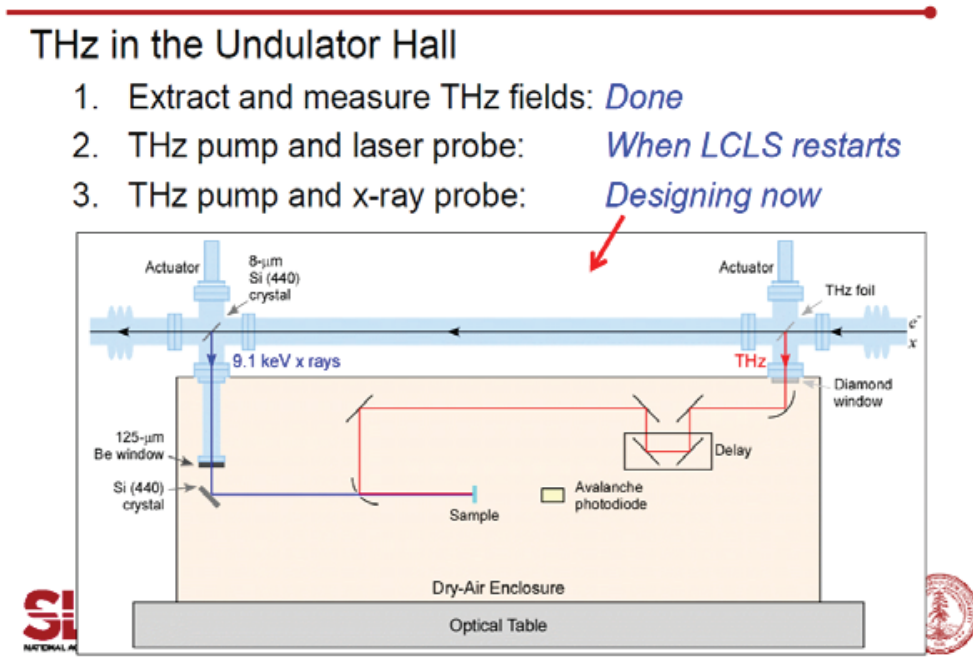

A.S. Fisher et. al., (2011) -> SLAC

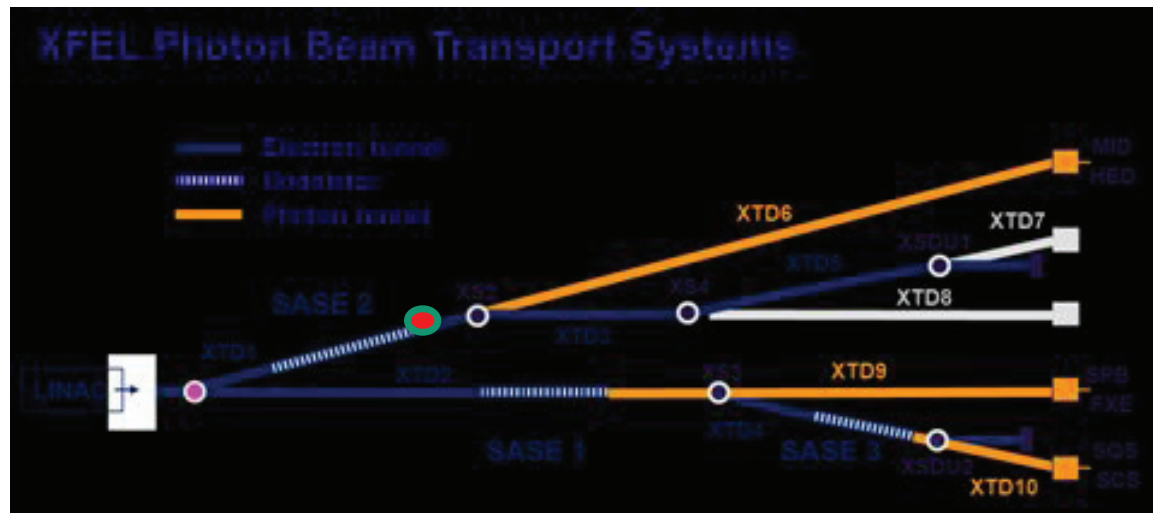

Proposal for European XFEL by N. Stojanovic (DESY), G. Geloni, T. Tschentscher (X-FEL $\mathrm{GmbH})$,T. Cowan, M. Gensch (HZDR)

Only limited number of experiments can be performed in the tunnel. What about separate (low energy) accelerator based IR/THz source for pumpprobe experiments? 


\section{Pump-probe experiments at $\mathrm{x}$-ray FEL user facility}

\section{Dedicated accelerator based IR/THz source}

- Electron accelerator (similar to PITZ): warm rf gun (up to $6 \mathrm{MeV}$ ) + warm accelerating section(s). With one (two) accelerating section electron energy is up to 25 (45) MeV. Bunch charge: from a fraction of $\mathrm{nC}$ to $4 \mathrm{nC}$

- Undulator: APPLE-II, period: $4 \mathrm{~cm}$, length 5 meters

- Radiation with wavelength below 200 um is generated by SASE FEL.

- Powerful coherent radiation with wavelength above 200 um is generated in the undulator by tailored (compressed) electron beam.

Properties of the radiation:

Example: operation at 100 um

- Wavelength range: $10 \mathrm{um}$... $1 \mathrm{~mm}$ (30 THz - $0.3 \mathrm{THz}$ )

- Radiation pulse energy: a few $100 \mathrm{uJ}$

- Peak power: 10 ... $100 \mathrm{MW}$

- Spectrum bandwidth 2 .. 3\%.
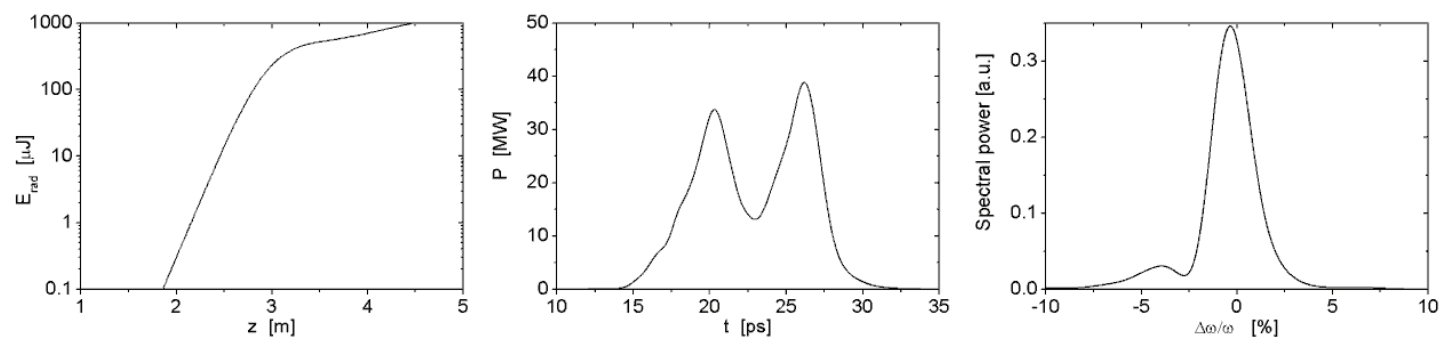

- Also installation of radiators for the production of a single-cycle radiation field using the mechanism of transition and edge radiation.

- Other types of diffraction radiators (e.g., Smith-Purcell, Cherenkov, etc.) can be implemented as well. 


\section{Pump-probe experiments at $\mathrm{x}$-ray FEL user facility}

Dedicated accelerator based IR/THz source

Potential to extend pump-probe experiments with femtosecond electron pulses.

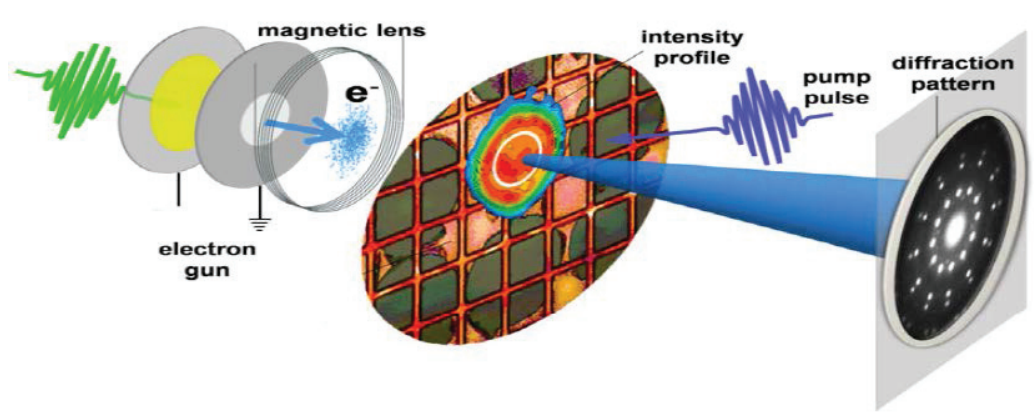

REGAE pump-probe (photon/electron) facility at CFEL/DESY

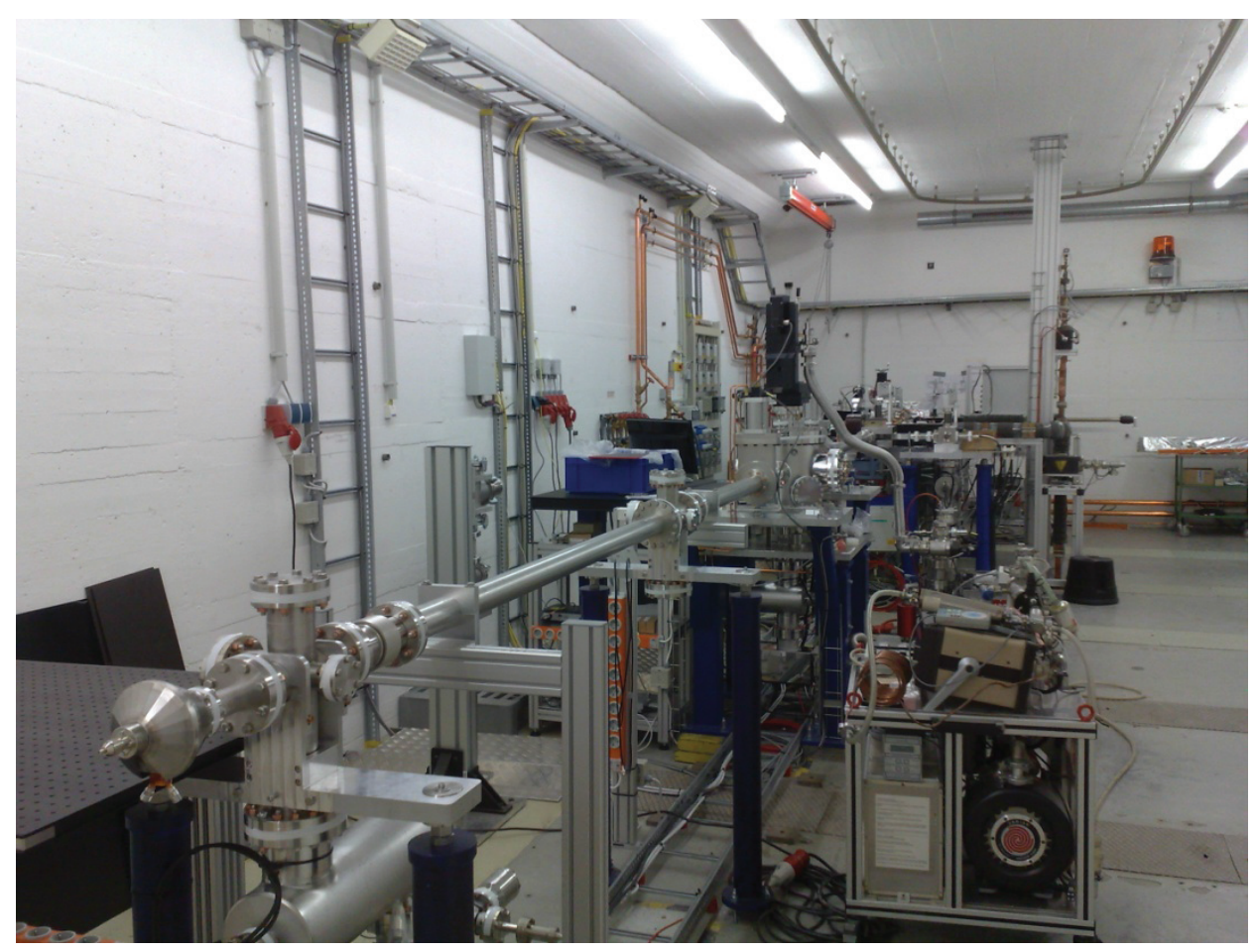




\section{Summary}

Нецмнотт

- Accelerator based IR/THz source meets all requirements for pump-probe experiments.

- Dimensions of the experimental hall $(25 \times 90 \mathrm{~m})$ are rather large, and it can accommodate such a source. Construction of a radiation shielded annex like present PITZ facility is also possible.

- Scale of IR/THz beamline is comparable with those operating at FLASH and TELBE.

- Prototype of the accelerator already exists - it is PITZ facility in Zeuthen.

- Can be excellent investment of efforts of accelerator consortium after finishing construction phase of the European XFEL.
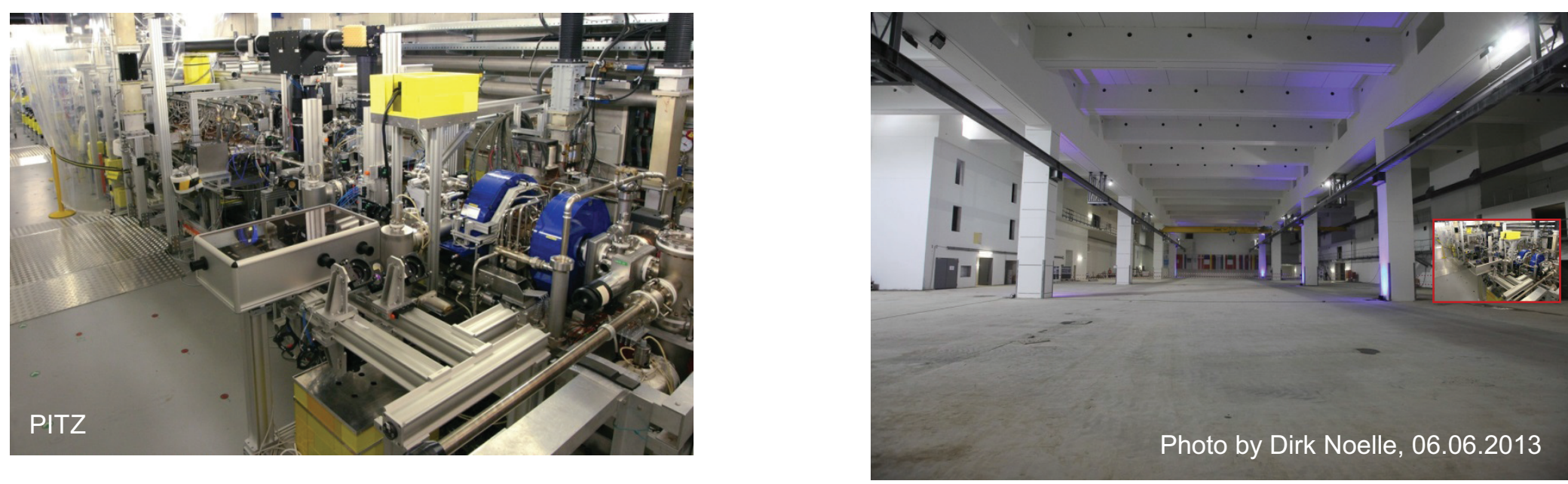

We are grateful to our colleagues from the European XFEL GmbH, CFEL, and DESY for many useful discussions: R. Brinkmann, A. Cavalieri, S. Molodtsov, J. Pflueger, N. Stojanovic, Th. Tschentscher, and many others. We are grateful to N. Mezentsev and K. Zolotorev (BINP, Novosibirsk) for providing us up-to-date information on the technology of superconducting magnets. 